\title{
Induction of interferon lambda in influenza a virus infected cells treated with shRNAs against M1 transcript
}

\author{
P. ŠVANČAROVÁ ${ }^{1}$, D. SVETLÍKOVÁ ${ }^{1}$, T. BETÁKOVÁ ${ }^{1,2^{*}}$ \\ ${ }^{1}$ Institute of Virology, Slovak Academy of Sciences, Dúbravská cesta 9, 84505 Bratislava, Slovak Republic; \\ ${ }^{2}$ Center for Molecular Medicine, Vlárska 3-7, 83101 Bratislava, Slovak Republic
}

Received September 25, 2014; accepted May 13, 2015

\begin{abstract}
Summary. - RNA interference (RNAi) represents a form of post-transcriptional gene silencing mediated by small interfering RNAs (siRNA) and provides a powerful tool to specifically inhibit viral infection. To investigate therapeutic capacity of siRNAs targeting $M$ gene, six vectors with U1-short hairpin RNA (shRNA) expression system were prepared and tested in infected cells and animals. In infected cells, three of six shRNAs targeting M1 gene significantly $(\mathrm{P}<0,01)$ reduced the virus titer to $66 \%, 45 \%$ or $21 \%$, respectively. Replication of IAV and levels of M1 RNAs were significantly reduced in the cells transfected with shRNAs, which decreased the virus titer. IFN- $\alpha / \beta$ altered in shRNAs-treated cells. The level of IFN- $\lambda$ (type III interferon) mRNA was significantly increased in the infected cells treated with shM22, shM349, shM522, and (type I interferon) as well as IP-10 (type II interferon) mRNAs were not significantly their mixtures. The increased level of IFN- $\lambda$ mRNA corresponded to significantly increased level of RIG-1 mRNA. shRNAs inhibited influenza virus infection in a gene-specific manner in co-operation with IFN- $\lambda$. Some constructs targeting the M1 transcript prolonged the survival of infected mice.
\end{abstract}

Keywords: influenza virus; shRNA; M1 protein

\section{Introduction}

The influenza A virus (IAV) belongs to the family Orthomyxoviridae. Its genome contains eight segments of negative sense, single-stranded RNA (vRNA), each encapsidated into ribonucleoprotein composed of the viral RNA-dependent RNA polymerase and multiple copies of the viral nucleoprotein. The approximately $13 \mathrm{~kb}$ genome encodes up to 16 proteins: $\mathrm{PB} 1, \mathrm{~PB} 2, \mathrm{PA}, \mathrm{NP}, \mathrm{HA}, \mathrm{NA}, \mathrm{M} 1$, M2, NS1, NEP, PB1-F2, PB1-N40, PA-X, PA-N155, PA-N182 and M42 (Wise et al., 2009, 2011, 2012; Jagger et al., 2012; Muramoto et al., 2013).

"Corresponding author. E-mail: virubeta@savba.sk; phone: +4212-59302440.

Abbreviations: $\mathrm{IAV}=$ influenza $\mathrm{A}$ virus; $\mathrm{RNAi}=\mathrm{RNA}$ interfer ence, $\operatorname{siRNA}(\mathrm{s})=$ small interfering $\mathrm{RNA}(\mathrm{s})$; $\operatorname{shRNA}(\mathrm{s})=$ small hairpin RNA(s)
The matrix protein $\mathrm{M} 1$ is a multifunctional protein playing many essential roles throughout the virus life cycle. M1 protein is a major structural protein, which associates with ribonucleoprotein and the cytoplasmic tails of hemagglutinin and neuraminidase (Harris et al., 2001). This protein plays key roles in controlling RNP trafficking and virion assembly. During the entry of the virus, the M1-RNP interaction must be disrupted to enable the transport of RNPs into the nucleus (Martin and Helenius, 1991; Bui et al., 1996). Following the late' synthesis of $\mathrm{M} 1$, some molecules enter the nucleus (Bucher et al., 1989) and interacts with RNPs. NEP links this complex with the cellular nuclear-export protein CRM1, which mediates RNP export (Neuman et al., 2000; Elton et al., 2001).

Influenza is a major cause of morbidity and mortality around the world. Available vaccines provide only partial protection and are ineffective against unexpected strains due to antigenic drift and antigenic shift. The therapeutic 
benefit of antiviral agents is limited by the drug resistance and antigenic changes. Therefore, new strategies for the prevention and treatment of IAV are currently being sought. RNAi represents a novel technology for inhibiting the replication of IAV.

RNAi comprises gene silencing mediated by short doublestranded RNAs which are known as siRNAs. Inside the cell, siRNAs associate with the RNA-induced silencing complex - RISC and the guide strand anneals to its complementary target mRNA and facilitates the cleavage by Argonaute 2, a core endonuclease of RISC (Grishok et al., 2001; Hutvagner et al., 2001; Ketting et al., 2001; Miyoshi et al., 2008; Sakurai et al., 2011). shRNA can be generated from plasmid or viral expression constructs and represents an alternative RNAi approach (Gobeil et al., 2008; Blakely et al., 2011; Betáková and Švančarová, 2013). shRNAs are expressed in the nucleus and associate with RISC only after being processed by two separate endonucleses, Drosha and Dicer (Kim and Rossi, 2009).

Conserved $\mathrm{M}$ gene provides a suitable target for IAV inhibition. Transfection of cells with some M1-specific siRNAs resulted in reduced replication of IAV (Ge et al., 2003, 2004; Hui et al., 2004; Zhou et al., 2007). In the present study, we demonstrated the effect of shRNAs targeting the $M$ gene on the treatment of an established IAV infection. The mRNA levels of M1, IFN- $\alpha$, IFN- $\beta$, IFN- $\gamma$, IFN- $\lambda$, and RIG-1 were compared in the cells. Five shRNAs targeting the M1 transcript and one shRNA targeting the transcriptional region common to both $\mathrm{M} 1$ and $\mathrm{M} 2$ were constructed and their potential to inhibit IAV in MDCK cells and mice were investigated.

\section{Materials and Methods}

Cells and viruses. MDCK (ATCC CCL) cells were grown in Dulbecco's modified Eagle's medium (DMEM) containing 10\% FCS. Influenza virus A/PR/8/34 was cultured in 10-day-old fertile hen's eggs.

Plasmid construction. The siRNAs targeting the M gene of $\mathrm{A} / \mathrm{PR} / 8 / 34$ virus were designed using siRNA Target Designer-Version 1.51 (Promega). The sequences and names of the constructs are listed in the Table 1. The M sequence represents a mutated sequence, which was used as a control. The oligonucleotides were cloned into the pGeneClip $^{\mathrm{TM}}$ vector using GeneClip ${ }^{\mathrm{TM}} \mathrm{U} 1$ Hairpin Cloning Systems (Promega) according to the manufacturer's instruction. The plasmids were purified using the PureYield Plasmid Miniprep System (Promega). The MDCK cells were transfected with the shRNAvectors using TransPass ${ }^{\mathrm{TM}} \mathrm{HeLa}$ Transfection Reagent (New England Biolabs) according to the manufacturer's protocol and transfection efficiacy was confirmed via fluorescence microscopy.

Fluorescence microscopy. MDCK cells were grown on glass coverslips and transfected with $2 \mu \mathrm{g}, 4 \mu \mathrm{g}, 8 \mu \mathrm{g}$ or $10 \mu \mathrm{g}$ of shRNA vector.
Twenty-four hours after transfection, the cells were fixed with $4 \%$ paraformaldehyde and viewed using an Olympus IX70 microscope. The images were captured using Silicon Graphics Delta vision.

Antiviral assay in MDCK cells. A confluent monolayer of MDCK cells in 24-well plates was infected with influenza $\mathrm{A} / \mathrm{PR} / 8 / 34$ virus at a multiplicity of infection (MOI) of 0.05 for $1 \mathrm{hr}$ at room temperature. After adsorption, the cells were washed with PBS and then cultured in serum-free DMEM containing trypsin $(2 \mu \mathrm{g} / \mathrm{ml})$ at $37^{\circ} \mathrm{C}$. After $16 \mathrm{hr}$, the infected cells were transfected with shRNA vectors using TransPass ${ }^{\mathrm{TM}} \mathrm{HeLa}$ Transfection Reagent (New England Biolabs) as described above. Four hours after transfection, the transfection medium was removed and the cells were incubated in DMEM containing trypsin $(2 \mu \mathrm{g} / \mathrm{ml})$ at $37^{\circ} \mathrm{C}$. Twenty-four hours post-transfection, the cells were scraped and centrifuged at $500 \mathrm{x} g$ for $2 \mathrm{~min}$. Viral titers in the supernatants were determined via plaque assay as described by Svetlikova (2010). RNA isolation. RNA from shRNA-transfected and infected cells was extracted using the SV Total RNA Isolation System (Promega). RNA yields were evaluated by spectrophotometer at $260 \mathrm{~nm}$. 400 ng of RNA was reverse transcribed using random hexa-nucleotide primers and MuLV reverse transcriptase (Finnzymes).

Semi-quantitative RT-PCR. To determine the presence of mRNA of IFN- $\alpha$, IFN- $\beta$, IP-10, IFN- $\lambda$, RIG- 1, M1, and $\beta$-actin, RT-PCR was performed. The primers targeting $\beta$-actin, IFN- $\beta$ and IP 10 (precursor IFN- $\gamma$ ) were described previously (Varga et al., 2011; Zhang et al., 2014). The sequences of the primers were as follows: 5'-CTT GGG ATG AGA CCC TCC TAG AC-3' (forward) and 5'-GCA CAA GGG CTG TAT TTC TTC TC-3' (reverse) for IFN- $\alpha$ of subtypes 1- and 2; 5'-CTG ACG CTG AAG GTT CTG GAG-3' (forward) and 5'-AAT TCA GGT CTC GCG TGA GGA GGC-3' (reverse) for IFN- $\lambda 2$ and 3 ; 5 '-TGC AGT CAC GTC TTA TGT GAT AG-3' (forward) and 5'-CTC CCA CTG CTC GAA CCA GAA AC-3' (reverse) for RIG-1; and 5'-CAA GAC CAA TCC TGT CAC CTC TG-3' (forward) and 5'-CAA AAT GAC CAT CGT CAA CAT C-3' (reverse) for M1. The intensity of the obtained PCR bands was determined by using Gene Tools image analysis software.

AIV infection and siRNA treatment of Balb/c mice. Mouse-adapted human influenza virus $\mathrm{A} / \mathrm{PR} / 8 / 34$ was prepared by passaging in mouse lungs. Animals were treated according to the European Union standards and the fundamental ethical principles including animal welfare requirements were respected. The experiments were performed under light anesthesia using zoletil $(50 \mathrm{mg} / \mathrm{kg})$. All animal experiments were evaluated and approved by the Permit Number: 4370/13-221 and 1204/11-221.

Groups $(n=8)$ of 5-week-old female BALB/c mice (Faculty of Medicine, Masaryk University, Czech Republic) weighing between 16 and $20 \mathrm{~g}$ were anesthetized and inoculated intranasally with $40 \mu \mathrm{l}$ of mouse-adapted A/PR/8/34 virus $\left(4 \times \mathrm{LD}_{50}\right)$. Sixteen hours post-infection, the mice were intravenously injected with $10 \mu \mathrm{g}$ of shRNA pre-incubated with TurboFect ${ }^{\mathrm{TM}}$ in vivo Transfection Reagent (Thermo Scientific) according to the manufacturer's protocol. Each evaluated group was separated into two subgroups by 4 animals. The mice in the first subgroup were weighed daily. The 
lungs of mice in the second subgroup were collected on the second day after transfection. Organ homogenates were prepared, and the level of IAV was determined via plaque assay.

Statistical analyses. Significant differences in the virus titer between the control group (untreated cells) and shRNA-transfected or infected cells were calculated using the unpaired Student's t-test. $P$ values $<0.05$ were considered significant. Statistical analysis was performed using Graph-Pad Prism software (http://www.graphpad. com/quickcalcs/ttest1.cfm).

\section{Results}

\section{Cloning and expression of shRNAs}

A total of 5 shRNAs (M112, M349, M408, M420, and M522) were chosen such that they covered the entire M1 transcript, and one shRNA (M22) was targeting the transcriptional region common to both $\mathrm{M} 1$ and $\mathrm{M} 2$ proteins. MDCK cells were transfected with different amounts of the shRNAs constructs and the transfection efficiency was confirmed via fluorescent microscopy. The maximal expression was detected when using 4-8 $\mu$ g of DNA (Fig. 1). Transfection with $10 \mu \mathrm{g}$ of DNA did not increase the number of transfected cells due to the high toxicity. Transfection efficiency in MDCK cells was comparable with transfection efficiency in HeLa cells (data not shown).

Inhibition of influenza virus replication by transient expression of single shRNA in MDCK cells

MDCK cells were infected with IAV for $16 \mathrm{hr}$ followed by transfection with shRNA. Infected MDCK cells transfected with the mutated plasmid (shM) served as a positive control. Different concentrations of shRNAs were tested to determine the maximal inhibitory effect. Constructs shM112, shM408, and shM420 did not affect the replication of IAV. shM22,
shM349, and shM522 significantly $(\mathrm{P}<0.01)$ reduced the virus titer to $66.3 \%, 45.2 \%$ or $21.1 \%$, respectively (Fig. 2 ). These constructs reached their maximal efficiency at the concentration of $8 \mu \mathrm{g}$ of DNA. Further increase of DNA concentration resulted in lower inhibition of virus replication due to toxicity (data not shown).

Inhibition of influenza virus replication by transient expression of multiple shRNAs in MDCK cells

None of the evaluated constructs fully inhibited the replication of IAV. Mixtures containing $1 \mu \mathrm{g}, 2 \mu \mathrm{g}$, or $3 \mu \mathrm{g}$ of each individual construct were applied to infected MDCK cells. The first mixture contained all six constructs and reduced the virus titer only to $47.5 \%$ (Fig. 3). Removing of construct shM22 from the mixture 2 resulted in a decrease of virus titer to $21,95 \%$ ( $\mathrm{P}<0.01)$. The third mixture, which contained constructs with statistically significant inhibitory effect, displayed lower inhibitory effect than single shM522 and decreased the virus titer to $36.3 \%$. The constructs shM112, shM408, and shM420, which individually did not influence the replication of IAV, resulted in statistically significant reduction of the virus titer to $56.5 \%(\mathrm{P}<0.05)$.

Reduction of mRNA specific to $M 1, R I G-1$ and interferons in shRNA transfected cells

To investigate whether shRNAs inhibited IAV replication in a gene-specific manner, cells were transfected with shRNAs. The amount of shRNA resulting in the highest inhibition was tested. At $24 \mathrm{hr}$ post-transfection, the cells were infected with IAV. Four hours later, cell lysates were used for RNA purification and the amount of mRNA specific to M1 transcript was determined. As seen in Fig. 4, treatment of cells with shM did not inhibit transcription of mRNA-M1. Treatment with shM522 reduced synthesis of mRNA-M1 to about $30 \%$. Consistent with the previous result, shRNAs with negative effect on replication of IAV significantly reduced the

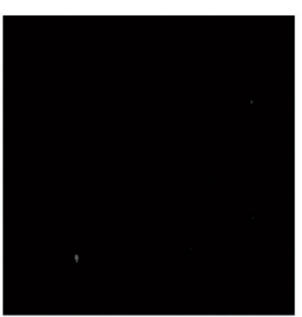

$0 \mu \mathrm{g}$

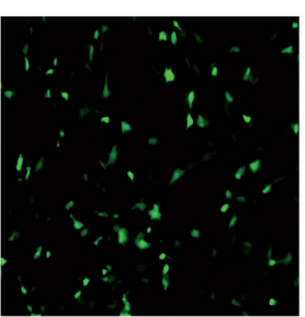

$2 \mu g$

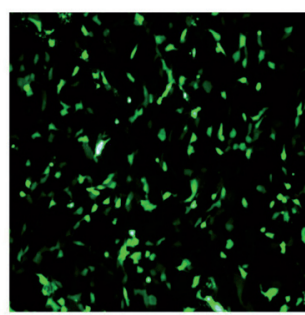

$4 \mu \mathrm{g}$

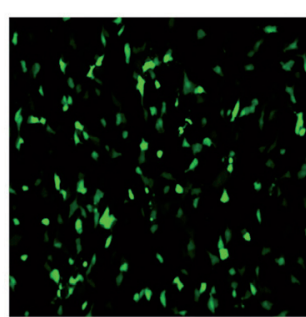

$8 \mu \mathrm{g}$

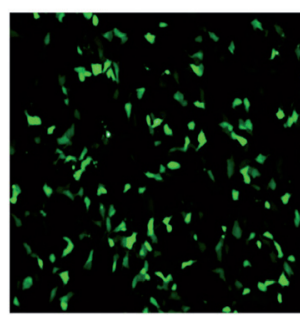

$10 \mu \mathrm{g}$

Fig. 1

Tranfection efficiency in MDCK cells

MDCK cells were transfected with $2 \mu \mathrm{g}, 4 \mu \mathrm{g}, 8 \mu \mathrm{g}$ and $10 \mu \mathrm{g}$, of shRNA as described in Materials and Methods. Expression of green fluorescent protein was evaluated by fluorescence microscopy (magnification 10x). 


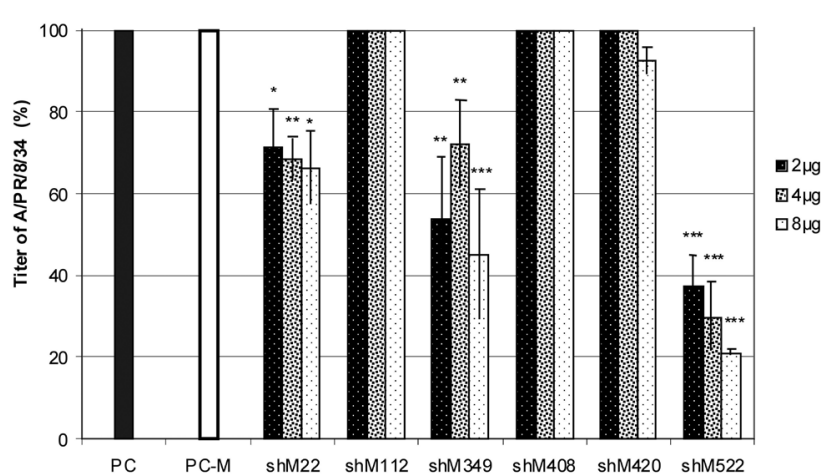

Fig. 2

Inhibition of influenza virus replication in MDCK cells by shRNAs targeting the M1 transcript

MDCK cells were infected with $\mathrm{A} / \mathrm{PR} / 8 / 34$, followed $16 \mathrm{hr}$ later by transfection with $2 \mu \mathrm{g}, 4 \mu \mathrm{g}$, or $8 \mu \mathrm{g}$ of shRNA. The virus titer was measured 24 $\mathrm{hr}$ after transfection. The column bars represent the average results with standard deviations from three independently performed experiments. A virus titer of $100 \%$ represents the level of infection of the MDCK cells transfected with the mutated plasmid (PC-M). Positive control (PC) represents MDCK cells infected with $\mathrm{A} / \mathrm{PR} / 8 / 34$ [H1N1]. Statistical significance $\left({ }^{\star} \mathrm{P}<0.05 ;{ }^{* *} \mathrm{P}<0.02 ;{ }^{* * *} \mathrm{P}<0.01\right)$ was determined using the unpaired Student's t-test.

amount of detected mRNA-M1. Cellular $\beta$-actin levels were not altered by virus infection and/or shRNAs transfection.

The IFN- $\alpha$, IFN- $\beta$, IP- 10 (precursor of IFN- $\gamma$ ) and IFN- $\lambda$ mRNAs were also analyzed. As seen in Fig. 4, levels of IFN- $\alpha / \beta$ (type I interferons), IP-10 (type II interferon) and IFN- $\lambda$ (type III interferon) mRNAs were not significantly changed in regard of negative or positive controls, respectively. The level of IFN- $\lambda$ mRNA was significantly increased in the infected cells treated with shM22, shM349, shM522, and with mixtures 2,3 , and 4 . The increased level of IFN- $\lambda$ mRNA corresponded to significantly increased level of RIG-1 mRNA.

\section{Inhibition of virus replication by shRNAs in Balb/c mice}

The activities of shRNAs in Balb/c mice were tested in two independent trials as described in Material and Methods.

The mice treated with the mutated plasmid shM and plasmids shM112 and shM408 died within 6 days (Fig. 5). Treatment with constructs shM522 and shM420 extended the survival of the mice by 1 or 2 days, respectively. Medication with shM22 or shM349 protected 1 of the 8 mice (Fig. 5). Morbidity was directly proportional to the weight loss (Fig. 6).

To study the synergic effect of the individual constructs, we prepared mixtures of shRNAs targeting the M1 transcript. Mixtures 1 and 2 consisted of all shRNAs and differed in construct shM22. While mixture 1 prolonged the survival of the mice by 2 days, the survival rate was

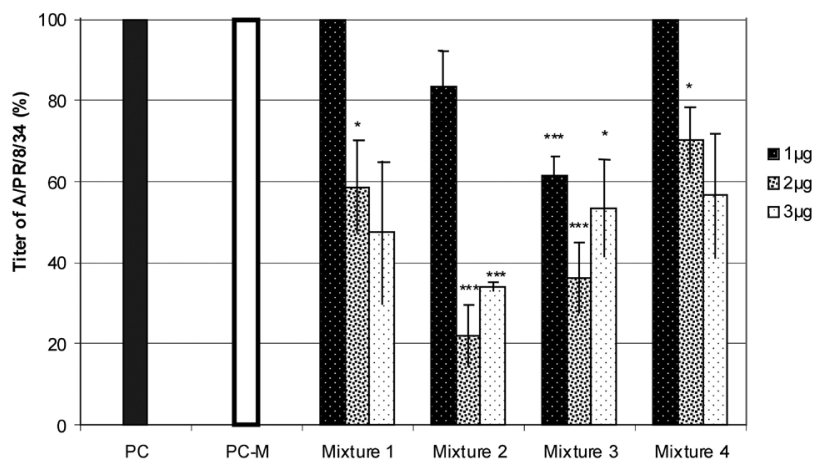

Fig. 3

Inhibition of influenza virus replication in MDCK cells by shRNA mixtures

MDCK cells were infected with $\mathrm{A} / \mathrm{PR} / 8 / 34$, followed $16 \mathrm{hr}$ later by transfection with a mixture containing $1 \mu \mathrm{g}, 2 \mu \mathrm{g}$ or $3 \mu \mathrm{g}$ of each shRNA construct. The virus titer was measured 24 hours after transfection. Mixture 1 consists of shM22, shM112, shM349, shM408, shM420, and shM522; mixture 2 consists of the same constructs as mixture 1 except for shM22; mixture 3 consists of shM22, shM349, and shM522; mixture 4 consists of shM112, shM408, and shM420. The column bars represent the average results with standard deviations from three independently performed experiments. A virus titer of $100 \%$ represents the level of infection of the MDCK cells transfected with the mutated plasmid (PC-M). Positive control (PC) represents MDCK cells infected with A/PR/8/34 [H1N1]. Statistical significance $\left({ }^{\star} \mathrm{P}<0.05\right.$; $\left.{ }^{* *} \mathrm{P}<0.02 ;{ }^{* *} \mathrm{P}<0.01\right)$ was determined using the unpaired Student's t-test.

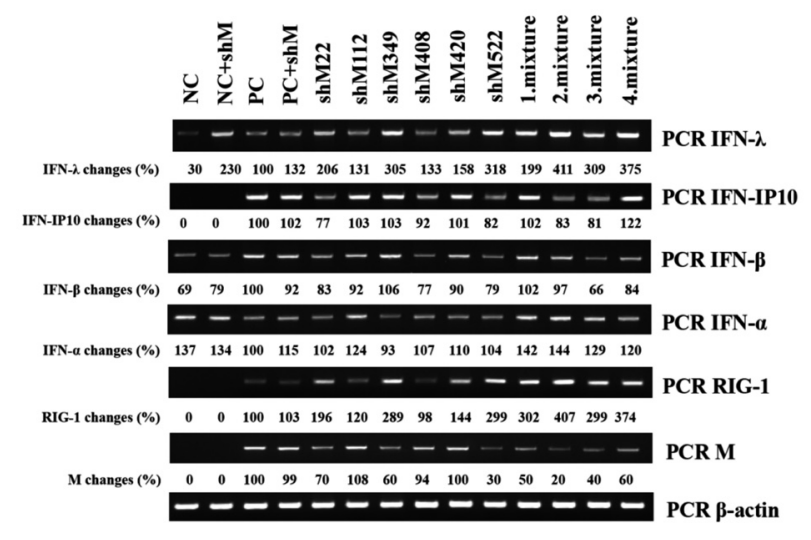

Fig. 4

Semi-quantitative RT-PCR analysis of M1, RIG-1 and IFN- $\alpha$, IFN- $\beta$, IFN- $\gamma$, and IFN- $\lambda$ mRNA in cells

The cells were transfected with shRNAs. At 24 hr post-transfection, the cells were infected with IAV. Four hours later, cell lysates were used for assessment of M1, RIG-1, IFN- $\alpha$, IFN- $\beta$, IFN- $\gamma$, and IFN- $\lambda$ mRNA as described in Materials and Methods.

slightly improved after removing construct shM22 from the mixture (Fig. 7). Mixture 3 comprised the shRNAs with statistically significant inhibitory effect. None of the mice were completely protected and their survival rate was not 


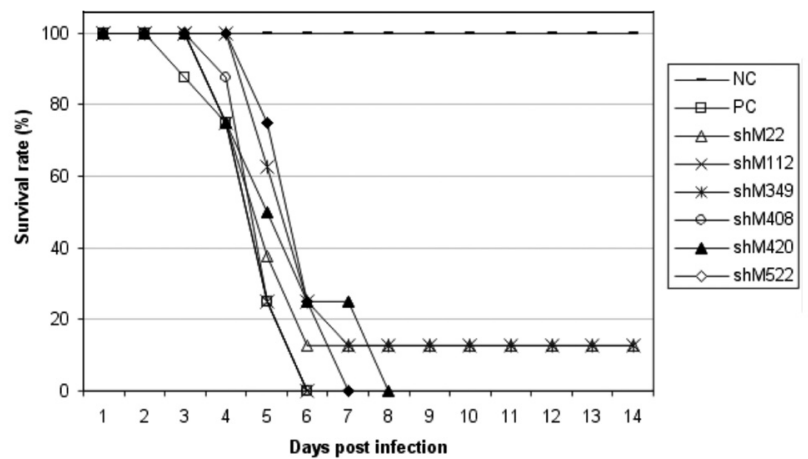

Fig. 5

Survival rate after treatment with shRNA against the M1 transcript $\mathrm{BALB} / \mathrm{c}$ mice were infected with $\mathrm{A} / \mathrm{PR} / 8 / 34\left(4 \mathrm{xLD}_{50}\right)$. After $16 \mathrm{hr}, 10 \mu \mathrm{g}$ of shRNA was intravenously administered to the mice. The protection rate is calculated as the number of surviving animals/total number of animals $\mathrm{x} 100 \%(\mathrm{n}=8)$ at each time point. NC represents mice injected with PBS, $\mathrm{PC}$ is positive control, infected mice treated with mutated plasmid.

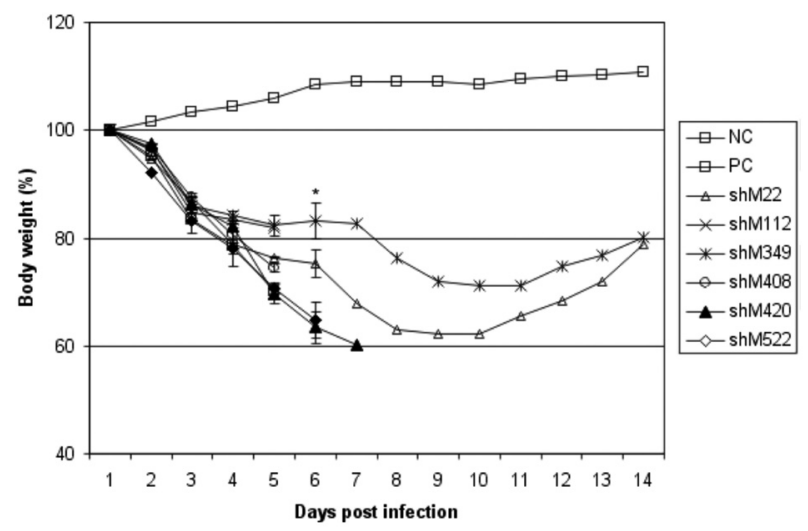

Fig. 6

The mean body weight \pm SD of mice that survived the infection after treatment with shRNA against M1 transcript

The values are the means of $2 \times 4$ mice per group. Statistical significance ${ }^{*} \mathrm{P}<0.05$. NC represents mice injected with PBS, PC is positive control, infected mice treated with mutated plasmid.

prolonged. On the other hand, administration of mixture 4 comprising constructs that did individually not influence the replication of IAV resulted in activity comparable with mixture 2 (Fig. 7 and Fig. 8).

\section{Discussion}

RNAi had been used as an effective antiviral strategy for its specific silencing of viral gene expression in cells and mice. The M1 protein appears to be a suitable target for siRNA due to its conservativeness. Most of these experiments were designed such that the cells were first transfected with a siRNA or shRNA, followed by infection with IAV 3-24 hr

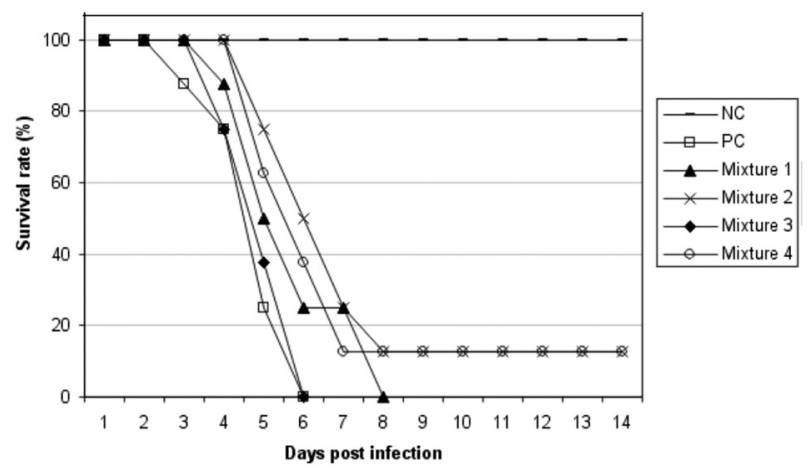

Fig. 7

Survival rate after treatment with shRNA mixtures

$\mathrm{BALB} / \mathrm{c}$ mice were infected with $\mathrm{A} / \mathrm{PR} / 8 / 34\left(4 \mathrm{xLD}_{50}\right)$. After $16 \mathrm{hr}$, mixtures of shRNAs (10 $\mu \mathrm{g}$ of each shRNA) were intravenously administered to the mice. Mixture 1 consists of shM22, shM112, shM349, shM408, shM420, and shM522; mixture 2 consists of the same constructs as mixture 1 except for shM522; mixture 3 consists of shM22, shM349, and shM22; mixture 4 consists of shM112, shM408, and shM420. NC represents mice injected with PBS, PC is positive control, infected mice treated with mutated plasmid. The protection rate was calculated as the number of surviving animals/total number of animals $\times 100 \%(n=2 \times 4)$ at each time point.

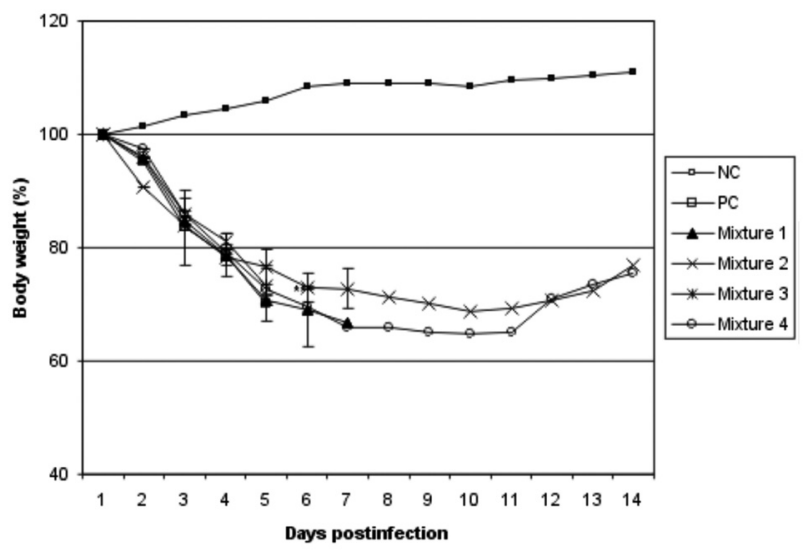

Fig. 8

The mean body weight \pm SD of the mice that survived the infection after treatment with mixtures of shRNAs

The values are the means of $2 \times 4$ mice per group. Statistical significance ${ }^{*} \mathrm{P}<0.05$. NC represents mice injected with PBS, PC is positive control, infected mice treated with mutated plasmid.

later (Ge et al., 2003; Hui et al., 2004; Zhou et al., 2004, 2007; Zhiqiang et al., 2010).

We were interested in the inhibition of virus replication in cells that were already infected. MDCK cells were infected with IAV for 16 hours and then were transfected with the shRNAs. Of six examined shRNAs targeting the M1 transcript, only three were able to inhibit the replication of IAV in MDCK cells. The construct shM349, identical with construct M-331 prepared by Hui et al. (2004), reduced the replication 
of IAV in MDCK cells and prolonged the survival of the infected mice. However, only $50 \%$ of our shRNAs targeting M1 transcript inhibited the replication of IAV. It is clear that the oligonucleotide sequence selected for the synthesis of the siRNAs plays an important role in their activity.

To improve the inhibition of virus replication, we decided to combine individual constructs and examine the effect of these mixtures on infected MDCK cells. It is interesting that the combination of all of the shRNAs targeting the M1 transcript, mixture 1, did not result in better inhibition than the individual shRNAs. On the contrary, mixture 2 (lacking shM22), reduced the virus titer to $21 \%$. Mixture 3, which contained constructs with statistically significant inhibitory effect, exhibited lower reduction of virus titer (36\%) than individual shRNAs. Positive synergic effect (56\%) was obvious when using mixture 4 , which consisted of individually negative shRNAs.

Two or more siRNAs can multiply (synergic effect) or inhibit (antagonistic effect) their individual efficiency, and this effect cannot be predicted. A combination of siRNAs targeting different regions of the human hepatitis $B$ virus exhibited improved inhibition of virus replication and antigen expression in a cell culture system (Chen et al., 2005). Similarly, partial silencing of viral transcripts by individual shRNAs impaired but did not block vesicular stomatitis virus replication, but the combination of these shRNAs into a multiple shRNA vector resulted in inhibition of viral replication (Ramirez-Carvajal and Long, 2012). McCaskill et al. (2013) reported opposing results, in which pre-treatment with individual siRNAs against hendra virus genes caused greater than $95 \%$ inhibition of mRNA expression, while combined pre-treatment resulted in 30-50\% silencing.

The inhibitory effect of our shRNAs was also evident when MDCK cells were at first transfected with shRNAs and then infected with IAV. Replication of IAV and levels of M1 RNAs were significantly reduced in cells transfected with shRNAs that decreased the virus titer. These results are in coincidence with previous findings of other authors and confirm that IAV replication is inhibited in a gene specific manner (Hui et al., 2004; Zhou et al., 2007; Švančarová et al., 2015).

Treatment of infected cells with mutated plasmid (shM) resulted in induction of IFN- $\lambda$ mRNA. shM, however, did not influence the transcription of IFN- $\alpha$, IFN- $\beta$, IP-10, and RIG-1 mRNA. Levels of IFN- $\alpha$, IFN- $\beta$ and IP-10 mRNAs in shRNAs treated cells oscillated around the control values. IFN- $\lambda$ mRNA was induced in infected cells treated with shM345, shM522 and mixtures 2, 3 and 4. The immune stimulation elicited by control shRNA and nonspecific therapeutic effects of shRNAs were already described by Robbins et al. (2008). However, in our case the immune stimulation by shM did not result in inhibition of IAV replication. Our results confirmed that IFN- $\lambda$ could play a critical role in the pathogenesis of IAV and IFN- $\lambda$ was induced through a RIG-1 -dependent pathway (Svetlikova et al., 2010; Okamoto et al., 2014; Wei et al., 2014). We conclude that shRNAs inhibited influenza virus infection in a gene-specific manner in cooperation with IFN- $\lambda$.

We examined the therapeutic effect of our shRNAs in mice infected for 16 hours. The shRNAs were administered intravenously. Some constructs targeting the M1 transcript prolonged the survival of infected mice. This protective effect did not correlate with the efficacy of these constructs in vitro. The efficacy of the individual constructs increased in the following order: shM $22<$ shM349 < shM522 in vitro. In vivo, the maximal protective effect was displayed by shM 22 and shM112. The survival time was prolonged, and $12.5 \%$ of the mice survived. A much worse protective effect was evident for shM522, which prolonged the survival of the

Table 1. Sequence of primers used for preparation shRNA constructs and their genomic position in IAV strain A/PR/8/34

\begin{tabular}{|c|c|c|}
\hline \multirow{2}{*}{ shRNA construct } & \multirow{2}{*}{ ORF position } & Forward primer \\
\hline & & Reverse primer \\
\hline \multirow{2}{*}{ M } & & 5'- TCT C AT TTG CAG GCC AAG AAC TTT CAA GAG AAG TTC TTG GTA TGC AAC CCC T -3' \\
\hline & & 5'- CTG CAG GGG TTG CAT ACC AAG AAC TTC TCT TGA AAG TTC TTG GCC TGC AAA T -3' \\
\hline \multirow{2}{*}{ M22 } & M gene & 5'- TCT CAA AGC AGG TAG ATA TTG ACT TCC TGT CAT CAA TAT CTA CCT GCT TTC C T -3' \\
\hline & $4-22 \mathrm{bp}$ & 5'- CTG CAG GAA AGC AGG TAG ATA TTG ATG ACA GGA AGT CAA TAT CTA CCT GCT TT -3’ \\
\hline \multirow{2}{*}{ M112 } & M gene & 5'- TCT CAT CGC ACA GAG ACT TGA ACT TCC TGT CAT TCA AGT CTC TGT GCG ATC C T -3' \\
\hline & $94-112 \mathrm{bp}$ & 5'- CTG CAG GAT CGC ACA GAG ACT TGA ATG ACA GGA AGT TCA AGT CTC TGT GCG AT -3' \\
\hline \multirow{2}{*}{ M349 } & M gene & 5'- TCT CCT CAA GAG GGA GAT AAC ACT TCC TGT CAT GTT ATC TCC CTC TTG AGC CT -3' \\
\hline & $331-349 \mathrm{bp}$ & 5'- CTG CAG GCT CAA GAG GGA GAT AAC ATG ACA GGA AGT GTT ATC TCC CTC TTG AG -3' \\
\hline \multirow{2}{*}{ M408 } & M gene & 5'- TCT CTG CAC TTG CCA GTT GTA TCT TCC TGT CAA TAC AAC TGG CAA GTG CAC C T -3' \\
\hline & $390-408 \mathrm{bp}$ & 5'- CTG CAG GTG CAC TTG CCA GTT GTA TTG ACA GGA AGA TAC AAC TGG CAA GTG CA -3' \\
\hline \multirow{2}{*}{ M420 } & M gene & 5'- TCT CTT GTA TGG GCC TCA TAT ACT TCC TGT CAT ATA TGA GGC CCA TAC AAC C T -3' \\
\hline & $402-420 \mathrm{bp}$ & 5'- CTG CAG GTT GTA TGG GCC TCA TAT ATG ACA GGA AGT ATA TGA GGC CCA TAC AA -3' \\
\hline \multirow{2}{*}{ M522 } & M gene & 5'- TCT CGT CTC ATA GGC AAA TGG TCT TCC TGT CAA CCA TTT GCC TAT GAG ACC CT -3' \\
\hline & $504-522$ bp & 5'- CTG CAG GGT CTC ATA GGC AAA TGG TTG ACA GGA AGA CCA TTT GCC TAT GAG AC -5' \\
\hline
\end{tabular}


mice by just one day. A synergistic effect was detected when using a combination of all constructs without shM22 and the combination of individually negative shRNAs. Treatment with the combination of all shRNAs prolonged the survival of the mice only for two days. Interestingly, the survival rate significantly increased after removing shM22 from mixture 1. One of the reasons for different combination effects as was seen in the case of shM22 can be explained by the possible competition of shRNA processing and RNAi machinery. The toxicity of shRNAs could be another possible reason for discrepancies between the activities of shRNAs in vitro and in vivo (Li et al., 2010; Tanudji et al., 2010; Loinger et al., 2012).

The therapeutic capacity of siRNAs targeting $M$ gene was investigated. Three of six shRNAs targeting M1 protein gene significantly $(\mathrm{P}<0.01)$ inhibited IAV replication in infected cells. Replication of IAV and levels of M1 RNAs were significantly reduced in the cells transfected with shRNAs that decreased the virus titer. Levels of IFN- $\alpha$, IFN- $\beta$ and IP-10 mRNAs in shRNAs-treated cells oscillated around the control values. IFN- $\lambda$ mRNA was significantly induced in infected cells treated with shRNAs that inhibited virus replication. Our data provide evidence that shRNAs targeting $M$ gene inhibited influenza virus infection in a gene-specific manner in co-operation with IFN- $\lambda$.

Acknowledgement. This research was supported by Slovak Research and Development Agency (grant No. APVV-0676-12) and by the VEGA-Grant Agency of Science (grant No. 2/0005/12).

\section{References}

Betáková T, Švančarová P (2013): Role and application of RNA interference in replication of influenza viruses. Acta Virol. 57, 97-104. http://dx.doi.org/10.4149/av $2013 \quad 0297$

Blakely K, Ketela T, Moffat J (2011): Pooled lentiviral shRNA screening for functional genomics in mammalian cells. Methods Mol. Biol. 781, 161-182. http://dx.doi.org/10.1007/9781-61779-276-2 9

Bucher D, Popple S, Baer M, Mikhail A, Gong YF, Whitaker C, Paoletti E, Judd A (1989): M protein (M1) of influenza virus: antigenic analysis and intracellular localization with monoclonal antibodies. J. Virol. 63, 3622-3633.

Bui M, Whittaker G, Helenius A (1996): Effect of M1 protein and low $\mathrm{pH}$ on nuclear transport of influenza virus ribonucleoproteins. J. Virol. 70, 8391-8401.

Chen Z, Xu ZF, Ye JJ, Yao HP, Zheng S, Ding JY (2005): Combination of small interfering RNAs mediates greater inhibition of human hepatitis B virus replication and antigen expression. J. Zhejiang Univ. Sci. B 6, 236-241. http://dx.doi. org/10.1631/jzus.2005.B0236

Elton D, Simpson-Holley M, Archer K, Medcalf L, Hallam R, McCauley J, Digard P (2001): Interaction of the influenza virus nucleoprotein with the cellular CRM1-mediated nuclear export pathway. J. Virol. 75, 408-419. http:// dx.doi.org/10.1128/JVI.75.1.408-419.2001

Ge Q, McManus MT, Nguyen T, Shen CH, Sharp PA, Eisen HN, Chen J (2003): RNA interference of influenza virus production by directly targeting mRNA for degradation and indirectly inhibiting all viral RNA transcription. Proc. Natl. Acad. Sci. USA 100, 2718-2723. http://dx.doi. org/10.1073/pnas.0437841100

Ge Q, Filip L, Bai A, Nguyen T, Eisen HN, Chen J (2004): Inhibition of influenza virus production in virus-infected mice by RNA interference. Proc. Natl. Acad. Sci. USA 101, 8676-8681. http://dx.doi.org/10.1073/pnas.0402486101

Grishok A, Pasquinelli AE, Conte D, Li N, Parrish S, Ha I, Baillie DL, Fire A, Ruvkun G, Mello CC (2001): Genes and mechanisms related to RNA interference regulate expression of the small temporal RNAs that control C. elegans developmental timing. Cell 106, 23-34. http://dx.doi. org/10.1016/S0092-8674(01)00431-7

Gobeil S, Zhu X, Doillon CJ, Green MR (2008): A genome-wide shRNA screen identifies GAS1 as a novel melanoma metastasis suppressor gene. Genes Dev. 22, 2932-2940. http://dx.doi.org/10.1101/gad.1714608

Harris A, Forouhar F, Qiu S, Sha B, Luo M (2001): The crystal structure of the influenza matrix protein M1 at neutral $\mathrm{pH}: \mathrm{M} 1-\mathrm{M} 1$ protein interfaces can rotate in the oligomeric structures of M1. Virology 289, 34-44. http://dx.doi. org/10.1006/viro.2001.1119

Hui EK, Yap EM, An DS, Chen IS, Nayak DP (2004): Inhibition of influenza virus matrix (M1) protein expression and virus replication by $\mathrm{U} 6$ promoter-driven and lentivirusmediated delivery of siRNA. J. Gen. Virol. 85, 1877-1884. http://dx.doi.org/10.1099/vir.0.79906-0

Hutvagner G, McLachlan J, Pasquinelli AE, Balint E, Tuschl T, Zamore PD (2001): A cellular function for the RNAinterference enzyme Dicer in the maturation of the let-7 small temporal RNA. Science 293, 834-838. http://dx.doi. org/10.1126/science.1062961

Jagger BW, Wise HM, Kash JC, Walters KA, Wills NM, Xiao YL, Dunfee RL, Schwartzman LM, Ozinsky A, Bell GL, Dalton RM, Lo A, Efstathiou S, Atkins JF, Firth AE, Taubenberger JK, Digard P (2012): An overlapping protein-coding region in influenza A virus segment 3 modulates the host response. Science 337, 199-204. http://dx.doi. org/10.1126/science.1222213

Ketting RF, Fischer SE, Bernstein E, Sijen T, Hannon GJ, Plasterk RH (2001): Dicer functions in RNA interference and in synthesis of small RNA involved in developmental timing in C. elegans. Genes Dev. 15, 2654-2659. http://dx.doi. org $/ 10.1101 /$ gad. 927801

Kim DH, Rossi JJ (2009): Transcriptional gene silencing using small RNAs. Methods Mol. Biol. 555, 119-125. http://dx.doi. org/10.1007/978-1-60327-295-7 9

Li X, Yoo JW, Lee JH, Hahn Y, Kim S, Lee DK (2010): Identification of sequence features that predict competition potency of siRNAs. Biochem. Biophys. Res. Commun. 398, 92-97. http://dx.doi.org/10.1016/j.bbrc.2010.06.041

Loinger,A. Shemla,Y. Simon I, Margalit H, Biham O (2012): Competition between small RNAs: a quantitative view. 
Biophys. J. 102, 1712-1721. http://dx.doi.org/10.1016/j. bpj.2012.01.058

Martin K, Helenius A (1991): Nuclear transport of influenza virus ribonucleoproteins: the viral matrix protein (M1) promotes export and inhibits import. Cell 67, 117-130. http:// dx.doi.org/10.1016/0092-8674(91)90576-K

McCaskill JL, Marsh GA, Monaghan P, Wang LF, Doran T, McMillan NA (2013): Potent inhibition of Hendra virus infection via RNA interference and poly I:C immune activation. PLoS One 8, e64360. http://dx.doi.org/10.1371/journal. pone. 0064360

Miyoshi K, Uejima H, Nagami-Okada T, Siomi H, Siomi MC (2008): In vitro RNA cleavage assay for Argonaute-family proteins. Methods Mol. Biol. 442, 29-43. http://dx.doi. org/10.1007/978-1-59745-191-8 3

Muramoto Y, Noda T, Kawakami E, Akkina R, Kawaoka Y (2013): Identification of novel influenza A virus proteins translated from PA mRNA. J. Virol. 87, 2455-2462. http:// dx.doi.org/10.1128/JVI.02656-12

Neumann G, Hughes MT, Kawaoka Y (2000): Influenza A virus NS2 protein mediates vRNP nuclear export through NESindependent interaction with hCRM1. EMBO J. 19, 67516758. http://dx.doi.org/10.1093/emboj/19.24.6751

Okamoto M, Oshiumi H, Azuma M, Kato N, Matsumoto M, Seya T (2014): IPS-1 is essential for type III IFN production by hepatocytes and dendritic cells in response to hepatitis C virus infection. J. Immunol. 192, 2770-2777. http:// dx.doi.org/10.4049/jimmunol.1301459

Ramirez-Carvajal L, Long CR (2012): Down-regulation of viral replication by lentiviral-mediated expression of shorthairpin RNAs against vesicular stomatitis virus ribonuclear complex genes. Antiviral. Res. 95, 150-158. http:// dx.doi.org/10.1016/j.antiviral.2012.05.007

Robbins M, Judge A, Ambegia E, Choi C, Yaworski E, Palmer L, McClintock K, MacLachlan I (2008): Misinterpreting the therapeutic effects of small interfering RNA caused by immune stimulation. Hum. Gene Ther. 19, 991-999. http://dx.doi.org/10.1089/hum.2008.131

Švančarová P, Svetlikova D, Betáková T (2015): Synergic and antagonistic effect of small hairpin RNAs targeting the NS gene of the influenza A virus in cells and mice. Virus Res. 195, 100-111. http://dx.doi.org/10.1016/j. virusres.2014.08.004

Svetlikova D, Kabat P, Ohradanova A, Pastorek J, Betáková $T$ (2010): Influenza A virus replication is inhibited in IFN- $\lambda 2$ and IFN- $\lambda 3$ transfected or stimulated cells. Antiviral. Res. 88, 329-333. http://dx.doi.org/10.1016/j. antiviral.2010.10.005

Tanudji M, Machalek D, Arndt GM, Rivory L (2010): Competition between siRNA duplexes: impact of RNA-induced silencing complex loading efficiency and comparison between conventional-21 bp and Dicer-substrate siRNAs.
Oligonucleotides 20, 27-32. http://dx.doi.org/10.1089/ oli.2009.0195

Varga ZT, Ramos I, Hai R, Schmolke M, García-Sastre A, Fernandez-Sesma A, Palese P (2011): The influenza virus protein PB1-F2 inhibits the induction of type I interferon at the level of the MAVS adaptor protein. PLoS Pathog. 7, e1002067. http://dx.doi.org/10.1371/journal. ppat. 1002067

Wei H, Wang S, Chen Q, Chen Y, Chi X, Zhang L, Huang S, Gao GF, Chen JL (2014): Suppression of interferon lambda signaling by SOCS-1 results in their excessive production during influenza virus infection. PLoS Pathog. 10, e1003845. http://dx.doi.org/10.1371/journal.ppat.1003845

Wise HM, Foeglein A, Sun J, Dalton RM, Patel S, Howard W, Anderson EC, Barclay WS, Digard P (2009): A complicated message: Identification of a novel PB1-related protein translated from influenza A virus segment 2 mRNA. J. Virol. 83, 8021-8031. http://dx.doi.org/10.1128/ JVI.00826-09

Wise HM, Barbezange C, Jagger BW, Dalton RM, Gog JR, Curran MD, Taubenberger JK, Anderson EC, Digard P (2011): Overlapping signals for translational regulation and packaging of influenza A virus segment 2. Nucleic Acids Res. 39, 7775-7790. http://dx.doi.org/10.1093/ nar/gkr487

Wise HM, Hutchinson EC, Jagger BW, Stuart AD, Kang ZH, Robb N, Schwartzman LM, Kash JC, Fodor E, Firth AE, Gog JR, Taubenberger JK, Digard P (2012): Identification of a novel splice variant form of the influenza A virus M2 ion channel with an antigenically distinct ectodomain. PLoS Pathog. 8, e1002998. http://dx.doi.org/10.1371/ journal.ppat.1002998

Zhiqiang W, Yaowu Y, Fan Y, Jian Y, Yongfeng H, Lina Z, Jianwei W, Qi J (2010): Effective siRNAs inhibit the replication of novel influenza A (H1N1) virus. Antiviral. Res. 85, 559561. http://dx.doi.org/10.1016/j.antiviral.2009.12.010

Zhou Y, Chan JH, Chan AY, Chak RK, Wong EY, Chye ML, Peiris JS, Poon LL, Lam E (2004): Transgenic plant-derived siRNAs can suppress propagation of influenza virus in mammalian cells. FEBS Lett. 577, 345-350. http://dx.doi. org/10.1016/j.febslet.2004.10.027

Zhang S, Sun Y, Chen H, DaY Y, Zhang Y, Yu S, Qiu X, Tan L, Song C, and Ding Ch (2014): Activation of the PKR/eIF2 $\alpha$ signaling cascade inhibits replication of Newcastle disease virus, Virology J. 11, 62 . http://dx.doi.org/10.1186/1743422X-11-62

Zhou H, Jin M, Yu Z, Xu X, Peng Y, Wu H, Liu J, Liu H, Cao S, Chen H (2007): Effective small interfering RNAs targeting matrix and nucleocapsid protein gene inhibit influenza A virus replication in cells and mice. Antiviral. Res. 76, 186-193. http://dx.doi.org/10.1016/j.antiviral.2007.07.002 